expected, inspiring confidence. But the vista of the unknown was far more interesting.

By searching and navigating through copious "see also" hyperlinks to other ELS entries (some are not yet linked) and using the "related articles" function, I traversed from bread-and-butter articles on the central dogma and RNA structure to principles of geological time and Aristotle, stopping by methanogenesis and brain stems en route. The essays are a digestible size, well written and frequently well illustrated. For those that need more explanation of terms, there is a glossary, although it seems somewhat lonely in its own window, and could perhaps be more usefully deployed by linking it directly to appropriate content.

If the ELS is disappointing in any way, it is with respect to some of the technical aspects of the website. Although the web pages are beautiful, bandwidth is still an issue for most of us, and heavy use of graphics does not assist fast downloads. This, in combination with other features such as the use of frames, several pop-up windows and an occasionally temperamental search engine, often makes the ELS feel slow and clunky.

But perhaps most important, the ELS has

made only minimal use of hypertext linking to other online resources. The links from article citations to their abstracts are sparse, even for very recent articles from wellknown journals that are certainly indexed by PubMed. Although stand-alone lists of links to useful websites are supplied, the few URLs cited in the articles are printed out and not hyperlinked.

Over and above these basic points are missed opportunities, for example, to link to database entries of genes discussed, author biographies, online teaching resources, or perhaps even to allow keyword-generated automatic searches of PubMed or the Nature journals.

Of course, with hyperlinks come maintenance issues, so perhaps these omissions were deliberate, or they are planned for the next phase of development. If so, then with some further engineering the ELS will graduate from being a useful stand-alone reference work to a true information portal, in the proud tradition of its ancestors.

Johanna McEntyre is at the National Center for Biotechnology Information, National Library of Medicine, National Institutes of Health, 45 Center Drive, Bethesda, Maryland 20892, USA.

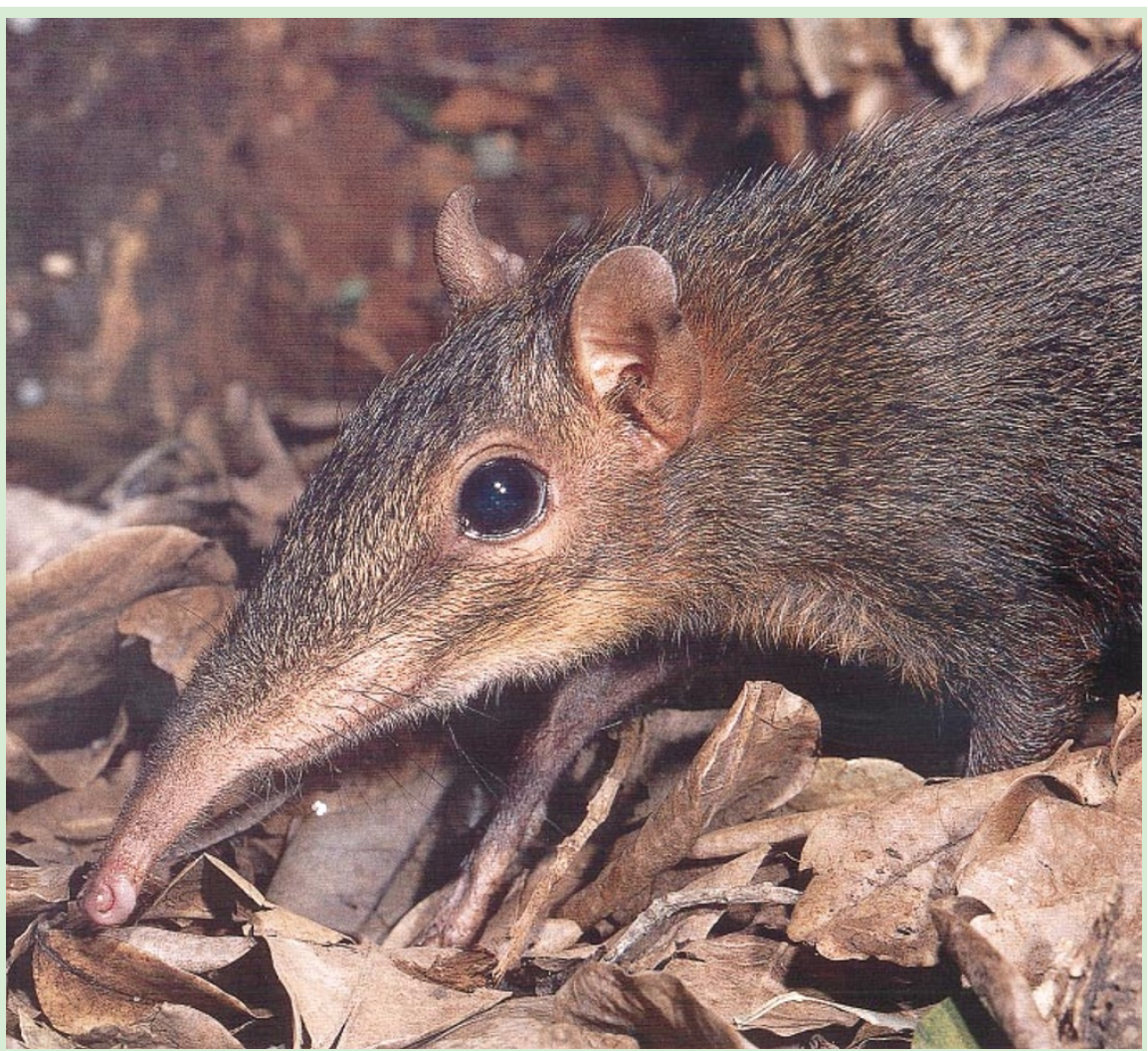

\title{
Poking around among the mammals
}

This melanistic variant of the checkered elephant shrew, Rhynchocyon cirnei, is just one of some 46,000 species catalogued and described in The New Encyclopedia of Mammals edited by David Macdonald (Oxford University

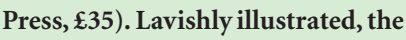
encyclopaedia is a completely updated revision of its 1984 predecessor. The conservation status and future prospects of each species are also documented.

\section{A new window on space}

\section{Revealing the Universe: The \\ Making of the Chandra X-ray Observatory}

by Wallace Tucker \& Karen Tucker

Harvard University Press: 2001. 296 pp.

$\$ 27.95$

\section{Ingo Lehmann}

Revealing the Universe tells the exciting story of the birth of X-ray astronomy and the development of the Chandra X-ray Observatory, which was launched by the Space Shuttle Columbia in 1999. The observatory, previously known as the Advanced $\mathrm{X}$-ray Astrophysics Facility, is one of NASA's four Great Observatories for space astrophysics, together with the Hubble Space Telescope, the Compton Gamma-Ray Observatory and the Space InfraRed Telescope Facility. Its unique imaging capability, high sensitivity and spectral resolution in the soft and hard X-ray wavebands enable us to investigate the nature of high-energy phenomena from such extraordinary objects as supernovae, neutron stars and black holes.

$\mathrm{X}$-ray photons have such high energies that they interact strongly with the Earth's atmosphere, making it impossible to use ground-based instruments for observing astronomical X-ray sources. In 1962, Riccardo Giacconi, Herbert Gursky and colleagues, using a simple X-ray detector carried on a rocket, discovered the first cosmic X-ray source, Sco X-1 (in the constellation Scorpius), and the cosmic X-ray background, opening up X-ray astronomy as a new window on the Universe.

The success of the Uhuru X-ray satellite in the early 1970s led to calls for a large $\mathrm{X}$-ray telescope that could provide data of almost the same quality as an optical telescope. Wallace and Karen Tucker describe the technological and political challenges from the first proposal for a large X-ray telescope in 1970 , to the launch of the Chandra X-ray Observatory some 30 years later. They offer an inside view on the efforts by many people, including Harvey Tananbaum, Giacconi, Martin Weisskopf and Charlie Pellerin, to implement this enormous NASA project.

The book focuses more on the story of Chandra than on astrophysics, but it does present an overview of the first impressive results from the observatory's mission. One minor shortcoming is the lack of a mention of Europe's recent contributions to X-ray astronomy, in particular those made by the XMM-Newton observatory.

Ingo Lehmann is at the Astrophysikalisches Institut Potsdam, An der Sternwarte 16,

14482 Potsdam, Germany. 